Article

\title{
Translation and Validation of the Mindful Self-Care Scale-Chinese Version: A Pilot Study
}

\author{
Ming Yu Claudia Wong \\ Department of Sport, Physical Education and Health, Hong Kong Baptist University, Hong Kong, China; \\ 18481795@life.hkbu.edu.hk
}

Received: 22 October 2020; Accepted: 24 November 2020; Published: 1 December 2020

\begin{abstract}
Background: Numerous research studies have investigated and identified the high prevalence of poor mental health status among the Chinese population, for example suffering from a high level of anxiety and depression. Moreover, the Chinese population are shown to have a conservative attitude towards mental health problems and a passive help-seeking behaviour when suffering from psychological distress. Therefore, it is important to assess the levels of self-care among the Chinese population, which is considered as the basis for tackling psychological well-being issues by the World Health Organization (WHO). Self-care was conceptualised and measured by the Mindful Self-Care Scale (MSCS), but without being translated and adapted to the Chinese population. The purpose of this study is to translate the scale into Chinese and to conduct a pilot test to validate the scale among the Chinese population. (2) Methods: Participants $(\mathrm{N}=161)$, with 96 females and 65 males, with an age range from 18 to 62 , mage $=26.1$ ( $\mathrm{SD}=6.64)$, were recruited from Hong Kong $(\mathrm{n}=63)$, Taiwan $(\mathrm{n}=44)$, and mainland China $(\mathrm{n}=54)$ using convenience sampling. (3) Results: The results show a satisfying internal consistency (Cronbach's $\alpha=0.915)$ without items that need to be deleted as well as an adequate validity, with Kaiser-Mayer-Olkin (KMO) value of 0.856 and significant correlation between self-care and the two variables: self-compassion and well-being $(r=0.403, r=0.172, p<0.001)$. (4) Conclusions: The results preliminarily indicate that the Chinese version of MSCS is reliable and valid. However, test-retest reliability and confirmatory factor analysis should be performed with a larger sample size for further validation.
\end{abstract}

Keywords: mindful self-care; measurement; mental health; mindfulness; psychometric properties; self-compassion

\section{Introduction}

According to the traditional Buddhism ideology, people tend to focus on showing love and mercy to people around them. Nevertheless, individuals rarely indicate love and kindness to themselves [1]. In other words, people seldom focus on their internal self and care for their individual psychological needs when experiencing negative emotions [2]. In particular, during the lockdown period of COVID-19, the mental status of Chinese people has also been "locked down" with little attention paid to their own well-being.

\subsection{Psychological Status of the Chinese Population}

Numerous research studies have investigated the prevalence of poor mental health status among the Chinese population. Studies have shown that more than half of the sample of Chinese individuals, mainly aged 18-24, tended to experience mental health problems [3-6]. Yet, their help-seeking behaviour showed a negative association with their mental health status, and hence individuals with poor mental health status had less willingness to seek help from mental health services [7]. Furthermore, the prevalence was shown to worsen due to the outbreak of COVID-19. 
In Hong Kong, The Hong Kong Jockey Club Centre for Suicide Research has surveyed the general public to highlight the potential psychological impact brought by the COVID-19 pandemic, especially the negative effects of the social-distancing measures on people's well-being. The survey showed nearly $40 \%$ and $70 \%$ of the participants were suffering from post-traumatic stress disorder and depression, respectively. Within the high depression rate, around $10 \%$ of the participants indicated suicidal ideation [8]. Although few studies have been conducted regarding the impact of COVID-19 on the Hong Kong population, Chinese scholars have sufficiently documented the effects of COVID-19 among the population of mainland China. In China, large-scale research indicated that almost $30 \%$ of participants were suffering from mild-to-moderate anxiety and a high level of depressive symptoms [9].

In addition, a large scale Chinese study, which involved 36 Chinese provinces, as well as other related regions including Hong Kong, Macau and Taiwan, was conducted utilising the COVID-19 Peritraumatic Distress Index (CPDI) to examine the effects of COVID-19, particularly on individuals' psychological status. The index involves the assessment of anxiety, depression, specific phobias, cognitive change, avoidance and compulsive behaviour, physical symptoms, and loss of social functioning [10]. Results indicate that more than one-third of the respondents suffered from psychological distress. Meanwhile, the severe levels of vulnerability and stress were associated with post-traumatic stress disorder and other mental health issues. Nonetheless, the research study demonstrated that people aged 18-30 were most likely to show a higher level of psychological distress compared to those aged 18 or below [11].

\subsection{Mindful Self-Care}

The Chinese population used to have a conservative attitude towards mental health problems, and they tended to hide such problems from the public [12]. Additionally, due to the COVID-19 pandemic, people in China were being restricted by the lockdown and stay-at-home orders, while Hong Kong and Taiwan citizens were encouraged to stay home and reduce social interaction. Hence, people were seen to be prevented from seeking external health help from friends, peers, or even mental health services.

According to the World Health Organization's (WHO) recommendation for the optimal mix of service [13], self-care is taken into account as the basis for tackling psychological well-being issues. Self-care refers to self-initiative behaviour that individuals adopt into their lives for better health and well-being [14]. By embracing self-care, individuals can avoid extensive reliance on others and assist the positive development of individuals' overall well-being. Studies have indicated that health-promoting self-care behaviours could significantly alleviate people's mental health problems, e.g., reducing perceived stress, anxiety, and depression, particularly among students and mental health professionals [15-17]. A healthy self can internally regulate self-esteem, stay calm, and self-soothe [18].

Self-care has been widely studied in both physical and mental health research. These research studies tend to summarise self-care behaviour with various other physical and psychological aspects, such as healthy eating, physical activity, self-regulation [19] and resilience. Yet, Cook-Cottone and Guyker (2018) [20] have conceptualised and defined mindful self-care, as well as comprehensively integrating the elements of self-care practices. Mindful self-care is defined as a person's mindful awareness towards their own internal psychological needs and external psychological demands. The intentional engagement in self-care practice could help respond to one's inner needs and hence enable positive psychological well-being [20]. According to Cook-Cottone's (2015) [21]. Attuned Model of Self, the internal self involves cognitive, emotional, and physiological aspects and yet could be affected by external factors including family, community and culture. Hence, Cook-Cottone and Guyker (2018) [20] expect mindful self-care practices to promote both the positive physiological and psychological status of individuals, as well as social well-being. Physiological well-being refers to physical self-care that involves a healthy diet, physical activities, and engagement in regular medical care. People with better physical care tend to enjoy positive physical health, and hence improve one's cognitive and psychological well-being [22]. The psychosocial aspects refer to self-care practices that involve 
mindful awareness, mindful relaxation, self-soothing, supportive relationships, self-compassion, and spiritual practices $[20,21]$. Specifically, self-compassion and mindfulness, as elements of mindful self-care that embrace warmth, love, and kindness to oneself, have also shown negative associations with vulnerability to mental health conditions such as depression and anxiety [2]. Further, engaging in mindful self-care contributes to a better quality of life as well as a higher level of health-related quality of life [23,24]. Therefore, by embracing mindful self-care, people are expected to be equipped with mindfulness, self-compassion, and a caring attitude towards their physical and psychological needs, which could reduce potential vulnerability and encourage help-seeking behaviour.

Based on the above literature review, the author hypothesized that cultivating mindful self-care could achieve a healthy self and reduce vulnerabilities among the Chinese population, especially during the COVID-19 pandemic. However, the Mindful Self-Care Scale (MSCS) [20] was newly developed in 2018; hence, it is important for the instrument to be translated into Chinese and be validated among the Chinese population. Therefore, this research study is aimed at conducting a pilot validation test on the translated Mindful Self-Care Scale (MSCS)—Chinese version—among the Chinese population.

\section{Materials and Methods}

\subsection{Translation and Validation Procedure(s)}

Forward and backward translations were performed on the Mindful Self-Care Scale between English and Chinese. The forward translation process involved two independent translators, with one translator that was not knowledgeable about the concept of the questionnaire and another with relevant background knowledge, in order to detect the possible differences within the translation. The backward translation was also conducted independently by another two translators to ensure the accuracy of the translation [25-27]. The translators are native English speakers, and the underlying concepts of the measures were unfamiliar to them to avoid biases. Any discrepancies between the translators were discussed and resolved.

Second, the initial version of the translation was first discussed among the translators and then given to five Chinese participants for content adjustment. After completing the above processes, the 5 participants were asked to verbally describe their understanding of each questionnaire item. This process enabled the researchers to ensure the translations are kept in line with the intention of the original scale. The final adjustment was made on the items with a need for revision to produce the pre-final version for the current pilot test. The original questionnaire (English version) are attached as a supplementary document at the end of the article (Table S1) [20].

\subsection{Data Collection}

The current pilot study targeted the general Chinese population, which included people aged 18 or above from China, Hong Kong, and Taiwan. The participants were recruited through convenience sampling. The data were collected through posting the online questionnaire invitations on different social media platforms, including Facebook, Instagram, and WeChat (for mainland China). The data collection started in early August and ended in mid-September when Hong Kong and most of mainland China were experiencing the second wave of the COVID-19 outbreak.

\subsection{Measures}

\subsubsection{Mindful Self-Care Scale (MSCS)}

The Mindful Self-Care Scale (MSCS) measures an individual's level of self-care across six subscales, including physical care, supportive relationship, mindful awareness, self-compassion and purpose, mindful relaxation, and supportive structure. It is rated with a 5-point Likert scale $(1=$ never, $2=$ rarely, $3=$ sometimes, $4=$ often, $5=$ regularly). The MSCS showed adequate reliability with a high total-item internal consistency (Cronbach's alpha $=0.89$ ), as well as a significant positive correlation with body 
esteem and negative correlation with eating disorder [20]. The Mindful Self-Care Scale (MSCS) also resulted in a satisfying validity with the Kaiser-Mayer-Oklin value being 0.860 ; and the confirmatory factor analysis of the six-factor model showing an acceptable index $\left(\chi^{2} / \mathrm{df}=3.02\right.$, Standardized Root Mean Square Residual $($ SRMR $)=0.071$, The Root Mean Square Error of Approximation $($ RMSEA $)=0.071$, and Comparative Fit Index $(\mathrm{CFI})=0.96)$.

\subsubsection{Self-Compassion}

The Chinese version of The Self-Compassion Scale-Short Form [28,29] was used to measure the level of self-compassion. The Self-Compassion Scale-Short Form consists of 12 items and the total score can determine the level of self-compassion of individuals. The 12 items of the scale are derived from the six self-compassion components, the same as the original scale: self-kindness, common humanity, mindfulness, self-judgment, isolation, and over-identification. The full version scale can be accessed through the self-compassion website developed by Kristin Neff, the originator of the Self-Compassion Scale. The items were rated on a 5-point scale $(1=$ almost never, $5=$ almost always $)$. The 12-item scale [29] showed an almost perfect correlation with the original 26-item scale. The 12-item Self-Compassion Scale's factor structure (Non-Normed Fit Index $(\mathrm{NNFI})=0.96 ; \mathrm{CFI}=0.97$ ) and Cronbach's alpha of 0.86 indicated it is a reliable measure of self-compassion. Furthermore, the Chinese Self-Compassion Scale-Short form showed an adequate and acceptable Cronbach's alpha value, test-retest reliability and construct validity among the Chinese population [28]. The alpha for the current sample was an acceptable internal consistency of 0.63 .

\subsubsection{Psychological Well-Being: The World Health Organization Five Well-Being Index (WHO-5) - Chinese Version}

The World Health Organisation Five Well-Being Index (WHO5) was used to measure the overall psychological well-being of the participants on a 6-point Likert scale $(0=$ at no time to $5=$ all of the time) [30]. The WHO5 is considered as a well-developed instrument to examine mental health status; it showed 0.89 scale sensitivity and 0.87 scale specificity on adolescents' depression screening test [31]. Additionally, the psychometric properties examination of the Chinese version revealed a 0.86 internal consistency and a moderate level of concurrent validity (0.41-0.51) [32]. Additionally, the internal consistency for the current sample was 0.70 , which verified the reliability and validity of the use of WHO5 among the Chinese population.

\subsection{Statistical Analysis}

The Cronbach's alpha value and the item-total score correlation, indicating the internal consistency (composite reliability), were used to evaluate the reliability of the questionnaire. Cronbach's alpha of at least 0.70 is expected to demonstrate the adequacy of the items' internal consistency. Secondly, with no items were deleted from the reliability test, the factorial validity of the questionnaire was determined by the Kaiser-Mayer-Olkin (KMO) value and Bartlett's test. A KMO value of at least 0.07 is expected to indicate an adequate, factorial, and valid questionnaire. Lastly, Pearson's correlation test and linear regression were conducted to assess the criterion validity of the translated Mindful Self-Care Scale by investigating the association among related variables as well as the expected psychological benefits of mindful self-care.

\section{Results}

A total of 161 Chinese adults participated in this mindful self-care scale validation survey, and the demographic information is demonstrated in Table 1 . The survey was conducted with no report of missing data. 
Table 1. Demographic information.

\begin{tabular}{ccccc}
\hline & & Mean (sd) & Frequency & Percentage (\%) \\
\hline \multirow{2}{*}{ Age } & & & \\
Gender & Male & & & \\
& Female & 65 & 40.1 \\
Geographic Location & & $96.63)$ & 59.3 \\
& Mainland China & 54 & 33.5 \\
& Hong Kong & 63 & 39.1 \\
& Taiwan & 44 & 27.3 \\
\hline
\end{tabular}

\subsection{Reliability}

The Cronbach's alpha value of the 36-item Mindful Self-Care Scale, including the three general items, was 0.93; while the 33-item scale had a Cronbach's alpha value of 0.92. The Cronbach's alpha values of the subscales were: mindful relaxation (0.74), physical care (0.78), self-compassion and purpose (0.78), supportive relationships (0.84), supportive structure (0.83) and mindful awareness (0.86). The statistical outcomes indicate that the Mindful Self-Care Scale achieved a satisfactory level of internal consistency. The Guttman split-half coefficient result (0.80) also suggested that adequate consistency was found between the first half and the second half of the scale. Furthermore, a significant low-to-moderate correlation was found between subscales ranging from $r=0.21-0.50$.

\subsection{Factorial Validity}

To ensure the factor distribution of the Chinese version of the MSCS is in-line with the original scale, the factor items were performed to verify the factorial validity. The factor analysis results revealed a Kaiser-Mayer-Olkin (KMO) value of 0.856 , which was similar to the original scale of 0.860 as well as a significant Bartlett's test of sphericity score, $\chi^{2}(630)=3066.22, p<0.001$. Hence, the results confirm that the common variance was adequate between items and thus showed an adequate factorial validity of the Mindful Self-Care Scale-Chinese version.

\subsection{Criterion Validity}

According to the above literature, higher level mindful self-care would be associated with a higher level of self-compassion and overall well-being. The mindful self-care score (Table 2) showed a significant positive moderate correlation with self-compassion, $r=0.39, p<0.001$ and a positive correlation with the WHO Five Well-Being Index, $r=0.16, p<0.001$, indicating an acceptable concurrent validity. Specifically, self-compassion had a high correlation coefficient with the mindful awareness subscale, social relation subscale and supportive structure subscale $(r=0.44,0.28,0.41, p<0.001)$. The WHO Five Well-Being Index showed a significant correlation with the social structure, mindful awareness subscale $(r=0.18,0.21, p<0.001)$ as well as the general items of the Mindful Self-Care Scale. While, only item five of the WHO Five Well-Being Index ("My daily life has been filled with things that interest me") showed significant correlation with all subscales of the Mindful Self-Care Scale, including the general items, ranging from $r=0.53$ to $0.24, p<0.001$. Additionally, self-compassion significantly predicted mindful self-care, with $\beta=0.23, p<0.001$, and thus indicating an acceptable predictive validity. 
Table 2. Correlation matrix for the Mindful Self-Care Scale, self-compassion and overall well-being.

\begin{tabular}{cccccccccc}
\hline & MSC & SCS & WHO & MR & PC & SC & SR & SS & MA \\
\hline SCS & $0.387^{* *}$ & - & & & & & & & \\
WHO & $0.159^{*}$ & $0.156^{* *}$ & - & & & & & \\
MR & $0.643^{* *}$ & $0.144^{*}$ & 0.04 & - & & & & \\
PC & $0.690^{* *}$ & $0.185^{* *}$ & 0.109 & $0.439^{* *}$ & - & & & \\
SC & $0.809^{* *}$ & $0.245^{* *}$ & 0.035 & $0.480^{* *}$ & $0.477^{* *}$ & - & & \\
SR & $0.766^{* *}$ & $0.277^{* *}$ & 0.092 & $0.373^{* *}$ & $0.372^{* *}$ & $0.595^{* *}$ & - & \\
SS & $0.8^{* *}$ & $0.408^{* *}$ & $0.184^{* *}$ & 0.3759 & $0.405^{* *}$ & $0.572^{* *}$ & $0.565^{* *}$ & - & \\
MA & $0.686^{* *}$ & $0.439^{* *}$ & $0.214^{* *}$ & $0.198^{* *}$ & $0.368^{* *}$ & $0.434^{* *}$ & $0.406^{* *}$ & $0.609^{* *}$ & - \\
\hline
\end{tabular}

Note: $\mathrm{MSC}=$ mindful self-care; SCS = Self-Compassion Scale; $\mathrm{WHO}=$ WHO Five Well-Being Index; $\mathrm{MR}=$ mindful relaxation subscale; $\mathrm{PC}=$ physical care subscale; $\mathrm{SC}=$ self-compassion and purpose subscale; $\mathrm{SR}=$ social relationship subscale; $\mathrm{SS}=$ supportive structure subscale; $\mathrm{MA}=$ mindful awareness subscale. ${ }^{*} p<0.05$; ${ }^{* *} p<0.01$.

\section{Discussion}

The present pilot study shows that the translated Mindful Self-Care Scale-Chinese version is reliable and valid to be applied to the Chinese population. The Mindful Self-Care Scale-Chinese version showed satisfactory internal consistency as well as a factorial validity comparable to that of the original scale. Moreover, it offered an adequate criterion validity with a moderate correlation and significant regression with self-compassion. Further, it showed a positive correlation with the WHO Five Well-Being index, which demonstrated the expected psychological effect of mindful self-care.

Based on the previous studies, self-compassion has been shown to be a self-care practice [33], which leads to better self-care ability [34]. In addition, self-care was promoted through self-compassion and mindfulness when dealing with psychological burnout and overwhelming emotions [35]. Hence, self-compassion, also an element of the mindful self-care scale, showed a significant positive correlation with mindful self-care, in-line with the existing literature [36]. In the present study, self-compassion showed the highest correlation with the social relationship and mindful awareness subscales. Perhaps this is due to the association between the elements within the social relationship and mindful awareness subscales and the two subscales of self-compassion: common humanity and mindfulness. Common humanity enables people to relate their own experiences with other people in the world and acknowledge that he or she is not the only person experiencing those deficiencies and failures [2]. Hence, individuals with a higher level of common humanity tend to seek help from or share their negative emotions with others, and thus cultivate a better social relationship. The mindfulness in self-compassion encourages people to be mindfully aware of their own thoughts and feelings rather than over-identifying them [2]. Hence, this component also corresponds with the mindful awareness subscale of the Mindful Self-Care Scale, which emphasises the awareness of one's feelings and thoughts with calmness. Furthermore, self-compassion showed a high correlation with self-regulation, especially with self-regulated health behaviours [37]; hence, it is also related to better organisation and supportive structure towards work and life, which can help maintain a balance between self needs and external demands.

It is worth noting that the current study was conducted around the second wave of the COVID-19 outbreak; hence, the research has especially highlighted the mental health situation of the Chinese population during COVID-19, while the present study also demonstrated the participants' level of self-compassion, well-being, and mindful self-care. Yet, they were all scored at a medium level, which indicates that the participants' mental health and well-being were not pleasurable enough.

To conclude, the results of the current pilot test on the relationship between mindful self-care and psychological well-being are in line with existing literature. Recent studies on mindful self-care have documented the positive effect on individuals' mental well-being, including reduced perceived stress [23] and predicted improvement in subjective well-being [38,39]. The current research has hence documented the significant relationship between mindful self-care and well-being, as well as 
demonstrating the validity of the Chinese version of Mindful Self-Care Scale with that of the WHO Five Well-Being Index. Therefore, the present study confirms the final version of the translated scale.

As for the limitations, the sample size of the pilot test was not sufficiently large enough for confirmatory factor analysis. Additionally, due to the usage of convenience sampling through the internet, only a limited number of participants were willing to disclose their contact information, making it difficult to conduct a test-retest reliability check. Additionally, the age coverage of this pilot test is considered as narrow, with a relatively young sample; hence, it could hardly be generalized to an older sample. Therefore, despite the present pilot study having verified the translated scale, a larger sample size and a randomized sampling across the age range will be needed for future research. Moreover, several studies have identified the possible barriers that prevent students from seeking help, which therefore lead to mental health problems. They reported concerns regarding privacy, the lack of emotional openness, as well as the lack of perceived need for help [40]. As a result, the self-understanding, self-care ability and help-seeking behaviour of children and adolescents are also considered as some of the essential factors affecting their mental health condition. Based on the outcomes of the current pilot test, the Chinese version of Mindful Self-Care Scale should be further validated in the Chinese children and adolescent population to investigate their level of mindful self-care and the association with positive psychological well-being.

Supplementary Materials: The following are available online at http://www.mdpi.com/2624-8611/2/4/25/s1, Table S1: The Mindful Self-care Scale.

Funding: This research received no external funding.

Acknowledgments: I would like to extend my thanks to my colleagues for their help in distributing the questionnaire on their social media for my data collection. Simon Wang at the Language Centre, Hong Kong Baptist University, has helped edit the manuscript.

Conflicts of Interest: The author declares no conflict of interest.

\section{References}

1. Pogosyan, M. Be Kind to Yourself: The Wisdom of Self-Compassion. Psychology Today. Available online: https:/www.psychologytoday.com/us/blog/between-cultures/201802/be-kind-yourself (accessed on 11 November 2020).

2. Neff, K. Self-compassion: An alternative conceptualisation of a healthy attitude toward oneself. Self Identity 2003, 2, 85-101. [CrossRef]

3. Fung, H.W.; Chung, H.M.; Ross, C.A. Demographic and mental health correlates of childhood emotional abuse and neglect in a Hong Kong sample. Child Abus. Negl. 2020, 99, 104288. [CrossRef] [PubMed]

4. Huang, R.; Ho, S.Y.; Wang, M.P.; Lo, W.S.; Lam, T.H. Reported alcohol drinking and mental health problems in Hong Kong Chinese adolescents. Drug Alcohol Depend. 2016, 164, 47-54. [CrossRef] [PubMed]

5. Liu, F.; Zhou, N.; Cao, H.; Fang, X.; Deng, L.; Chen, W.; Zhao, H. Chinese college freshmen's mental health problems and their subsequent help-seeking behaviors: A cohort design (2005-2011). PLoS ONE 2017, 12, e0185531. [CrossRef] [PubMed]

6. Zeng, W.; Chen, R.; Wang, X.; Zhang, Q.; Deng, W. Prevalence of mental health problems among medical students in china: A meta-analysis. Medicine 2019, 98, e15337. [CrossRef]

7. Chen, H.; Fang, X.; Liu, C.; Hu, W.; Lan, J.; Deng, L. Associations among the number of mental health problems, stigma, and seeking help from psychological services: A path analysis model among Chinese adolescents. Child. Youth Serv. Rev. 2014, 44, 356-362. [CrossRef]

8. Yip, P.S.F.; Chau, P.H. Physical Distancing and Emotional Closeness amidst COVID-19. Crisis J. Crisis Interv. Suicide Prev. 2020, 41, 153-155. [CrossRef]

9. Cao, W.; Fang, Z.; Hou, G.; Han, M.; Xu, X.; Dong, J.; Zheng, J. The psychological impact of the COVID-19 epidemic on college students in China. Psychiatry Res. 2020, 287, 112934. [CrossRef]

10. Qiu, J.; Shen, B.; Zhao, M.; Wang, Z.; Xie, B.; Xu, Y. A nationwide survey of psychological distress among Chinese people in the COVID-19 epidemic: Implications and policy recommendations. Gen. Psychiatry 2020, 33, e100213. [CrossRef] 
11. Sareen, J.; Erickson, J.; Medved, M.I.; Asmundson, G.J.G.; Enns, M.W.; Stein, M.; Leslie, W.; Doupe, M.; Logsetty, S. Risk factors for post-injury mental health problems. Depress. Anxiety 2013, 30, 321-327. [CrossRef]

12. Chong, S.A.; Verma, S.; Vaingankar, J.A.; Chan, Y.H.; Wong, L.Y.; Heng, B.H. Perception of the public towards the mentally ill in developed Asian country. Soc. Psychiatry Psychiatr. Epidemiol. 2007, 42, 734-739. [CrossRef]

13. World Health Organization. The Optimal Mix of Services; WHO: Geneva, Switzerland, 2007; Available online: https://www.who.int/mental_health/policy/services/2_Optimal\%20Mix\%20of\%20Services_ Infosheet.pdf (accessed on 13 August 2020).

14. Bickley, J.B. Care for the caregivers: The art of self-care. Semin. Perioper. Nurs. 1998, 7, 114-121. [PubMed]

15. Di Benedetto, M.; Towt, C.J.; Jackson, M.L. A cluster analysis of sleep quality, self-care behaviors, and mental health risk in Australian university students. Behav. Sleep Med. 2020, 18, 309-320. [CrossRef] [PubMed]

16. Rudaz, M.; Twohig, M.P.; Ong, C.W.; Levin, M.E. Mindfulness and acceptance-based trainings for fostering self-care and reducing stress in mental health professionals: A systematic review. J. Context. Behav. Sci. 2017, 6, 380-390. [CrossRef]

17. White, M.A.; Whittaker, S.D.; Gores, A.M.; Allswede, D. Evaluation of a Self-Care Intervention to Improve Student Mental Health Administered through a Distance-Learning Course. Am. J. Health Educ. 2019, 50, 213-224. [CrossRef]

18. Baker, H.S.; Baker, M.N. Heinz Kohut's self psychology: An overview. Am. J. Psychiatry 1987, 144, 1-9. [PubMed]

19. Tang, T.S.; Brown, M.B.; Funnell, M.M.; Anderson, R.M. Social support, quality of life, and self-care behaviors among African Americans with type 2 diabetes. Diabetes Educ. 2008, 34, 266-276. [CrossRef]

20. Cook-Cottone, C.P.; Guyker, W.M. The development and validation of the Mindful Self-Care Scale (MSCS): An assessment of practices that support positive embodiment. Mindfulness 2018, 9, 161-175. [CrossRef]

21. Cook-Cottone, C.P. Incorporating positive body image into the treatment of eating disorders: A model for attunement and mindful self-care. Body Image 2015, 14, 158-167. [CrossRef]

22. Hopkins, M.E.; Davis, F.C.; Van Tieghem, M.R.; Whalen, P.J.; Bucci, D.J. Differential effects of acute and regular physical exercise on cognition and affect. Neuroscience 2012, 215, 59-68. [CrossRef]

23. Depner, R.M.; Cook-Cottone, C.P.; Kim, S. Structural relationship between mindful self-care, meaning made, and palliative worker's quality of life. Int. J. Stress Manag. 2020. [CrossRef]

24. Feng, X.; Mosimah, C.I.; Sizemore, G.; Goyat, R.; Dwibedi, N. Impact of mindful self-care and perceived stress on the health related quality of life among young-adult students in west virginia. J. Hum. Behav. Soc. Environ. Self-Care Vicarious Trauma 2019, 29, 26-36. [CrossRef]

25. Beaton, D.; Bombardier, C.; Guillemin, F.; Ferraz, M.B. Recommendations for the cross-cultural adaptation of the DASH \& QuickDASH outcome measures. Inst. Work Health 2007, 1, 1-45.

26. Guillemin, F.; Bombardier, C.; Beaton, D. Cross-cultural adaptation of health-related quality of life measures: Literature review and proposed guidelines. J. Clin. Epidemiol. 1993, 46, 1417-1432. [CrossRef]

27. Tsang, S.; Royse, C.; Terkawi, A. Guidelines for developing, translating, and validating a questionnaire in perioperative and pain medicine. Saudi J. Anaesth. 2017, 11, S80-S89. [CrossRef] [PubMed]

28. Meng, R.; Yu, Y.; Chai, S.; Luo, X.; Gong, B.; Liu, B.; Yu, C. Examining psychometric properties and measurement invariance of a chinese version of the self-compassion scale-short form (SCS-SF) in nursing students and medical workers. Psychol. Res. Behav. Manag. 2019, 12, 793-809. [CrossRef] [PubMed]

29. Raes, F.; Pommier, E.; Neff, K.D.; Van Gucht, D. Construction and factorial validation of a short form of the self-compassion scale. Clin. Psychol. Psychother. 2011, 18, 250-255. [CrossRef]

30. Bech, P. Measuring the dimension of psychological general well-being by the WHO 5. Qual. Life Newsl. 2004, 32, 15-16.

31. Allgaier, A.K.; Pietsch, K.; Frühe, B.; Prast, E.; Sigl-Glöckner, J.; Schulte-Körne, G. Depression in pediatric care: Is the WHO-Five Well-Being Index a valid screening instrument for children and adolescents? Gen. Hosp. Psychiatry 2012, 34, 234-241. [CrossRef] [PubMed]

32. Kong, C.L.; Lee, C.C.; Ip, Y.C.; Chow, L.P.; Leung, C.H.; Lam, Y.C. Validation of the Hong Kong Cantonese version of World Health Organization five well-being index for people with severe mental illness. East Asian Arch. Psychiatry 2016, 26, 18-21.

33. Nelson, J.R.; Hall, B.S.; Anderson, J.L.; Birtles, C.; Hemming, L. Self-Compassion as self-care: A simple and effective tool for counselor educators and counseling students. J. Creat. Ment. Health 2017, 13, 121-133. [CrossRef] 
34. Mills, J.; Wand, T.; Fraser, J.A. Examining self-care, self-compassion and compassion for others: A cross-sectional survey of palliative care nurses and doctors. Int. J. Palliat. Nurs. 2018, 24, 4-11. [CrossRef] [PubMed]

35. Coaston, S.C. Self-care through self-compassion: A balm for burnout. Prof. Couns. 2017, 7, 285-297. [CrossRef]

36. Slatyer, S.; Craigie, M.; Heritage, B.; Davis, S.; Rees, C. Evaluating the Effectiveness of a Brief Mindful Self-Care and Resiliency (MSCR) Intervention for Nurses: A Controlled Trial. Mindfulness 2018, 9, 534-546. [CrossRef]

37. Biber, D.D.; Ellis, R. The effect of self-compassion on the self-regulation of health behaviors: A systematic review. J. Health Psychol. 2017, 24, 2060-2071. [CrossRef] [PubMed]

38. Chatterjee, S.; Jethwani, J. A study of The Relationship between Mindful Self-Care and Subjective Well-Being among College Students and Working Professionals. Int. J. Innov. Res. Technol. 2020, 7. [CrossRef]

39. Sünbül, Z.A.; Malkoç, A.; Gördesli, M.A.; Arslan, R.; Çekici, F. Mindful self-care dimensions as agents of well-being for students in mental health fields. Eur. J. Educ. Stud. 2018, 5. [CrossRef]

40. Hunt, J.; Eisenberg, D. Mental health problems and help-seeking behavior among college students. J. Adolesc. Health 2010, 46, 3-10. [CrossRef]

Publisher's Note: MDPI stays neutral with regard to jurisdictional claims in published maps and institutional affiliations.

(C) 2020 by the author. Licensee MDPI, Basel, Switzerland. This article is an open access article distributed under the terms and conditions of the Creative Commons Attribution (CC BY) license (http://creativecommons.org/licenses/by/4.0/). 